\title{
Estoque e Capacidade de Retenção Hídrica da Serrapilheira Acumulada na Restauração Florestal de Áreas Perturbadas na Mata Atlântica
}

\author{
Felipe Araujo Mateus ${ }^{1}$, Cristiana do Couto Miranda ${ }^{2}$, Ricardo Valcarcel ${ }^{1}$, \\ Pablo Hugo Alves Figueiredo ${ }^{1}$
}

\author{
${ }^{1}$ Departamento de Ciências Ambientais - DCA, Universidade Federal Rural do Rio de Janeiro - UFRRJ, \\ Seropédica/RJ, Brasil \\ ${ }^{2}$ Instituto Federal do Rio de Janeiro - IFRJ, Pinheiral/RJ, Brasil
}

\begin{abstract}
RESUMO
A serrapilheira, em restauração florestal espontânea, pode influir no balanço hídrico e em seus processos ecológicos. Neste estudo, foi avaliado o estoque de serrapilheira acumulada e sua retenção hídrica ex situ, pelo método de Blow, em locais similares em quatro níveis de restauração florestal espontânea, desenvolvidos a partir da espécie espontânea Clidemia urceolata DC., nos seguintes ambientes: pastagem abandonada (S1); capoeira (S2); capoeirão (S3), e fragmento florestal (S4). O amostrador $(0,25 \times 0,25 \times 0,05 \mathrm{~m})$ foi disposto aleatoriamente em 12 amostras por tratamento, durante o período de estiagem. O maior estoque de serrapilheira acumulada foi observado em S4 (5,15 Mg.ha-1) e a maior retenção hídrica, em S2 (343,87\%) e S3 (339,94\%), evidenciando que biomassa e diversidade de espécies condicionam a produção, porém não afetam as respostas hídricas da serrapilheira, cujas maiores retenções estão vinculadas à presença de material decíduo da espécie facilitadora nas fases pouco evoluídas e as menores retenções, nas fases mais evoluídas (30 anos de restauração), aumentando a umidade do solo.
\end{abstract}

Palavras-chave: balanço hídrico, serviços ecossistêmicos, facilitação.

\section{Stock and Water Retention Capacity of Litter Accumulated on the Forest Restoration of Disturbed Areas in the Atlantic Rainforest}

\begin{abstract}
Litter on spontaneous forest restoration can influence water balance and its ecological processes. In this study, we evaluated the stock of accumulated litter and its ex situ water retention by the Blow method in similar locations, at 4 levels of forest restoration spontaneously developed from the species Clidemia urceolata DC. in the following environments: abandoned pasture (S1), farmyard (S2), brushwood (S3), and forest fragment (S4). A sampler $(0.25 \times 0.25 \times 0.05 \mathrm{~m})$ was randomly prepared in 12 samples per treatment during the dry period. The largest stock of accumulated litter was observed in $\mathrm{S} 4\left(5.15 \mathrm{Mg} \mathrm{ha}^{-1}\right)$ and the greatest water retention levels were found in S2 (343.87\%) and S3 (339,94\%), demonstrating that biomass and species diversity influence production but do not affect the responses of litter water, whose greater retention levels are related to the presence of deciduous material from facilitative species in the poorly evolved phases and to lower retention levels in the more advanced stages (30 years of restoration), increasing soil moisture contents.
\end{abstract}

Keywords: water balance, ecosystem services, facilitation. 


\section{INTRODUÇÃO}

Regiões tropicais frágeis, onde os ciclos econômicos extrativistas, como exploração florestal, cafeicultura e pecuária leiteira e de corte, ocorreram no passado, gerando esgotamento dos solos e perda de serviços ecossistêmicos. O cenário atual do trecho fluminense do Médio Paraíba do Sul apresenta pastagens de baixa produtividade, com intensos e distintos processos erosivos (Menezes, 2008). Os poucos fragmentos florestais encontramse pulverizados em meio às extensas pastagens perturbadas, com diferentes níveis de degradação (Miranda et al., 2011). Áreas com mínima resiliência apresentam regeneração capaz de iniciar processos de restauração florestal espontânea (Carvalho, 2000; Miranda et al., 2011).

A restauração florestal em solos com traços de matéria orgânica, próximos a pequenos fragmentos florestais, requer estratégias específicas, em que o sinergismo dos seus serviços ecossistêmicos pode influir nos seus processos (Valcarcel \& Silva, 2000). Segundo os mesmos autores, essas estratégias requerem conhecimento autoecológico das espécies espontâneas e capacidade de combinar efeitos de resistência às adversidades locais (ataque de insetos, estresses hídrico e térmico, fogo, pastoreio extensivo e pouca oferta de propágulos) com facilitação, por essas espécies oferecidas.

A serrapilheira é um compartimento do ecossistema que apresenta implicações hidroecológicas nos processos de sucessão, pois o material vegetal depositado na superfície do solo, tais como folhas, cascas, ramos, material reprodutivo, flores, inflorescências, frutos, sementes e fragmento vegetal não identificável (Cianciaruso et al., 2006) pode regular a administração das chuvas e o lavado de nutrientes para o interior do solo, atuando desde o recebimento dos propágulos até sua germinação e desenvolvimento. A biomassa, em estágios de decomposição distintos, representa fonte de energia para as espécies (Brun et al., 2001), conferindo estabilidade ao sistema; também, juntamente com o solo, a biomassa controla processos fundamentais na dinâmica dos ecossistemas, como o desenvolvimento de espécies e a liberação de nutrientes (Pires et al., 2006), e a retenção de umidade (Vallejo, 1982).

A serrapilheira, além de ser fonte de energia e nutrientes para o solo, abriga fauna e microrganismos decompositores do ecossistema (Facelli \& Facelli, 1993), melhorando suas condições físicas (Mitchell \& Tell, 1977), facilitando a infiltração (Coelho Netto, 2003), reduzindo processos erosivos e atenuando o impacto das gotas de chuva (Facelli \& Pickett, 1991).

O acúmulo da serrapilheira no solo depende de alguns fatores: tipo de vegetação, nível sucessional, latitude, altitude, temperatura, ventos, precipitação, herbivoria, disponibilidade hídrica e estoque de nutrientes no solo (Facelli \& Picket, 1991; Portes et al., 1996). A produção de serrapilheira em florestas em fase inicial de sucessão, nas áreas tropicais úmidas, pode ser superior à das florestas maduras, pois apresentam espécies decíduas e composição florística diversificada (Meguro et al., 1979). Dessa forma, a taxa de acumulação de serrapilheira no período de maior crescimento do povoamento pode constituir um diferencial na restauração florestal espontânea, justamente quando as plantas mais necessitam de nutrientes e serviços ecossistêmicos, para facilitar o ingresso de novas espécies ambientalmente mais exigentes (Gonçalves et al., 2003).

Segundo Voigt \& Walsh (1976), a retenção de umidade pela serrapilheira acumulada está relacionada com os fenômenos de absorção e adsorção ou adesão superficial. A absorção depende, principalmente, de alguns aspectos: porosidade do material depositado, velocidade de decomposição, variação da precipitação e temperaturas do ambiente. A adsorção depende de: área das folhas, estrutura, relevo, forma, relação superfície/peso seco e composição orgânica.

Nesse contexto, faz-se necessário ampliar os conhecimentos sobre núcleos de vegetação regenerantes espontaneamente em pastagens perturbadas e suas implicações na dinâmica do acúmulo e da retenção hídrica da serrapilheira. O presente trabalho objetivou quantificar o estoque de serrapilheira acumulada e sua capacidade de retenção hídrica em quatro ambientes com diferentes níveis de restauração florestal, a partir do desenvolvimento espontâneo da espécie facilitadora Clidemia urceolata DC., conhecida popularmente como mixirico. 


\section{MATERIAL E MÉTODOS}

\section{1. Área de estudo}

O local de estudo se encontra na Bacia Hidrográfica do Ribeirão Cachimbal, (latitude $22^{\circ}$ $29^{\prime} 03^{\prime \prime}$ e $22^{\circ} 35^{\prime} 27^{\prime \prime}$ S, e longitude de $45^{\circ} 54^{\prime} 49^{\prime \prime}$ e $44^{\circ} 04^{\prime} 05^{\prime \prime} \mathrm{W}$ ). A parte experimental foi realizada na área do Instituto Federal do Rio de Janeiro (IFRJ), Campus Nilo Peçanha, município de Pinheiral-RJ (Figura 1).

O clima, segundo classificação de Köppen, é Am tropical, com inverno seco e verão chuvoso. A temperatura média anual está em torno de $20,9^{\circ} \mathrm{C}$, com máxima de $33{ }^{\circ} \mathrm{C}$, em dezembro e janeiro, e mínima de $12{ }^{\circ} \mathrm{C}$, em julho. A precipitação anual está em torno de $1.322 \mathrm{~mm}$ ano $^{-1}$, apresentando excedente entre dezembro e março, e escassez hídrica entre junho e setembro (INMET, 1992)

A vegetação pertence ao domínio do bioma Mata Atlântica, cuja cobertura original é Floresta Estacional Semidescidual Submontana (IBGE, 2012). No entanto, em função dos usos pretéritos, as áreas perturbadas são constituídas por pastagens de baixa produtividade, entremeadas por pequenos fragmentos florestais em fases iniciais de sucessão (Toledo \& Pereira, 2004).

\subsection{Amostragem}

Foram selecionadas quatro áreas similares (declividade média de 55\%, feição côncava predominante, altitude média de $418 \mathrm{~m}$, face de exposição sul-sudoeste), localizadas no terço médio

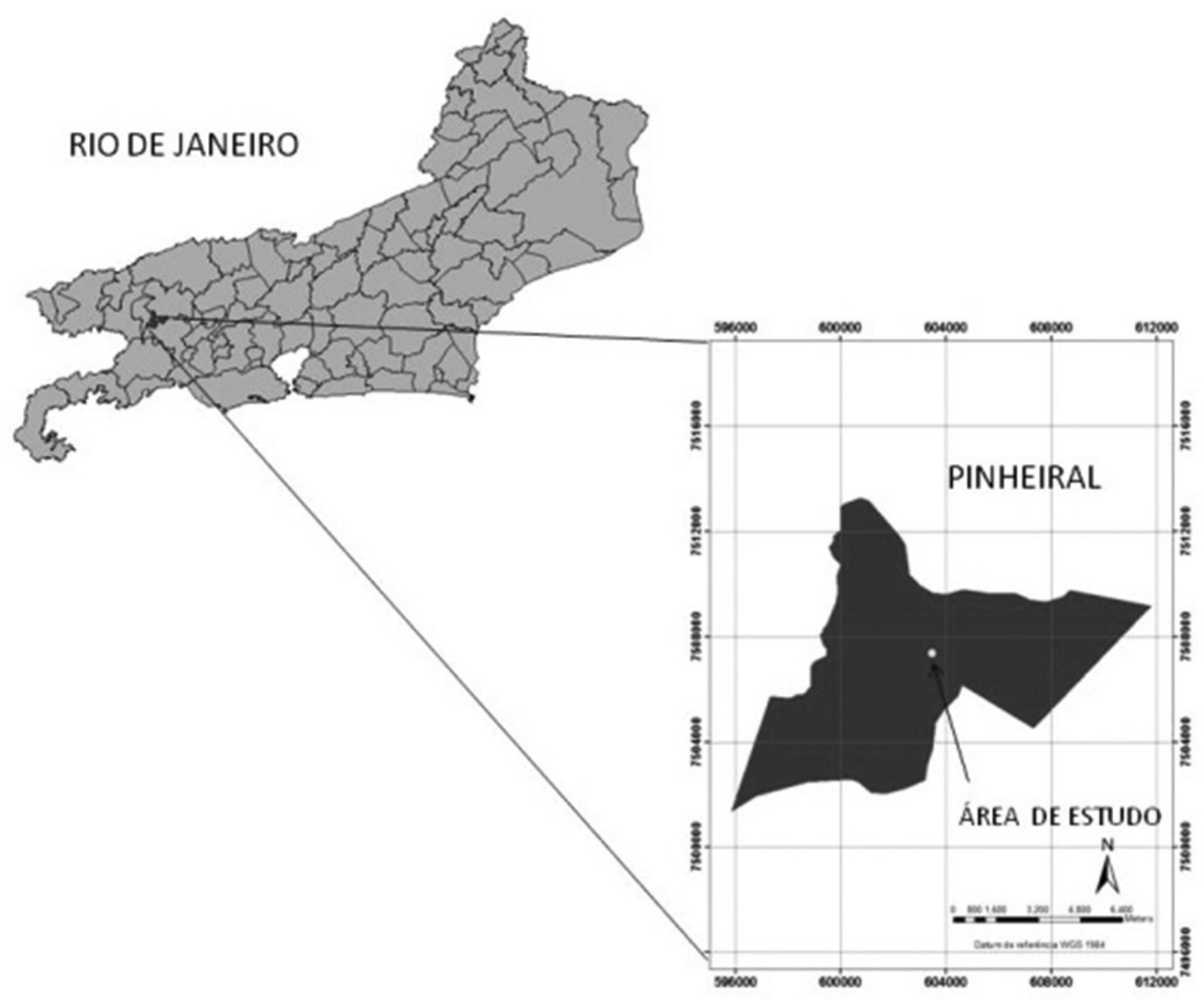

Figura 1. Área de estudo localizada no Instituto Federal do Rio de Janeiro (IFRJ), Campus Nilo Peçanha em Pinheiral-RJ.

Figure 1. Study area located at the Federal Institute of Rio de Janeiro (IFRJ), Nilo Peçanha Campus in Pinheiral - RJ. 
da topossequência, sobre Latossolos Amarelos Distróficos.

As áreas apresentam os seguintes níveis sucessionais: Sítio 1 (S1) - pastagem perturbada e abandonada (0,2 ha), com solo exaurido após pastoreio extensivo e fogo frequente, não sendo observadas espécies arbustivo-arbóreas com diâmetro à altura do peito maior ou igual a $5 \mathrm{~cm}$ (DAP), mas apresentando colonização inicial de Clidemia urceolata, com poucos indivíduos jovens e idade presumida de seis anos (Miranda, 2012); Sítio 2 (S2) - capoeira com dominância fitofisionômica de Clidemia urceolata e presença de espécies arbustivoarbóreas [Handroanthus ochraceus (Cham.) Mattos; Machaerium hirtum (Vell.) Stellfeld; Psidium guineense Sw.; Rapanea ferruginea (Ruiz \& Pav.) Mez], com aproximadamente 0,024 ha e idade presumida de dez anos (Miranda, 2012); Sítio 3 (S3) - capoeirão com dominância fitofisionômica de Clidemia urceolata e Cecropia pachystachya, sendo observados alguns indivíduos da espécie Casearia sylvestris Sw., com aproximadamente 0,024 ha e idade presumida de 15 anos (Miranda, 2012); Sítio 4 (S4) - fragmento florestal com aproximadamente 1 ha, composto por espécies pioneiras e secundárias iniciais [Siparuna guianensis Aubl.; Cecropia pachystachya Trécul; Sparattosperma leucanthum (Vel.) K. Schum; Schinus terebinthifolius Raddi; Casearia sylvestris Sw.; Eugenia cf. florida DC.; Astrocaryum aculeatissimum (Schott) Burret; Casearia sp.; Guarea guidonia (L.) Sleumer; Miconia prasina (Sw.) DC.] e caracterizado por estratos diferenciados (ervas, arbustos e árvores de pequeno porte) e grande quantidade de lianas; sua idade presumida é de 30 anos (Miranda, 2012; Figueiredo et al., 2011).

A quantidade de serrapilheira acumulada e sua capacidade de retenção hídrica foram avaliadas a partir de 12 amostras aleatoriamente coletadas por sítio amostral, em agosto de 2010. Utilizou-se coletor $(25 \times 25 \times 5 \mathrm{~cm})$ disposto sobre o solo, sendo a serrapilheira acondicionada em recipiente térmico e levado para o Laboratório de Manejo de Bacias Hidrográficas da UFRRJ.

O material orgânico foi limpo de sedimentos edáficos e seco em estufa a $70{ }^{\circ} \mathrm{C}$, até adquirir peso constante. Os dados médios de massa de serrapilheira acumulada total foram apresentados em Mg.ha-1 .

A capacidade de retenção hídrica $(\mathrm{CRH})$ da serrapilheira foi obtida a partir do Método de Blow
(1955), em que as amostras foram submersas em água durante 90 minutos, retiradas e depositadas em bandejas com $30 \%$ de declividade, para que drenassem naturalmente por 30 minutos. Posteriormente, as amostras foram pesadas em balança $(0,001 \mathrm{~g})$ $\left(\mathrm{MU}=\right.$ massa úmida - g) e levadas à estufa a $70{ }^{\circ} \mathrm{C}$, até adquirir peso constante ( $\mathrm{MS}=$ massa seca $-\mathrm{g}$ ), quando se determinou a capacidade de retenção hídrica (CRH) (Equação 1).

$$
\mathrm{CRH}(\%)=[(\mathrm{MU}-\mathrm{MS}) \div \mathrm{MS}] \times 100
$$

Os tratamentos foram avaliados a partir do teste de homogeneidade (Teste de Bartlett) e de normalidade (Teste de Lilliefors), para posterior análise de variância e teste de Tukey a 5\%. Todos os testes foram processados no programa Systat 11.0 (ZAR, 1999).

\section{RESULTADOS E DISCUSSÃO}

\subsection{Serrapilheira acumulada}

O fragmento florestal (S4) apresenta maior diversidade de espécies, com 30 anos de processos de restauração florestal espontânea, havendo apresentado maior média da serrapilheira acumulada $\left(5,15 \mathrm{Mg} \mathrm{ha}^{-1} \pm 1,51\right)(\mathrm{P}<0,001$ e $\mathrm{F}=10,83)$ do que os tratamentos S1 e S2, embora estatisticamente não tenha diferido do tratamento S3 (Figura 2).

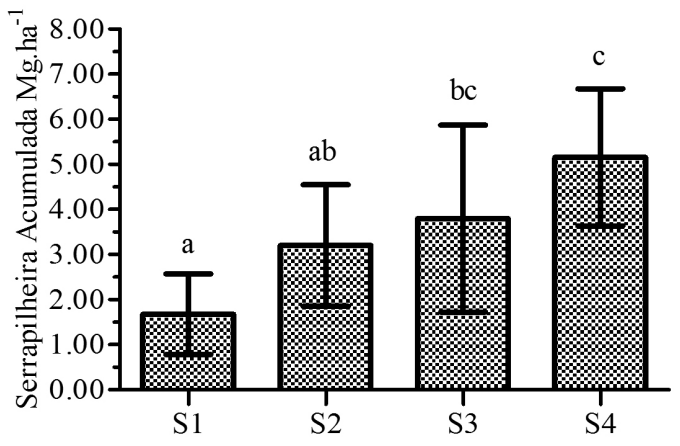

Figura 2. Médias da serrapilheira acumulada $\left(\mathrm{Mg} \cdot \mathrm{ha}^{-1}\right)$ e seus respectivos desvios padrões nos quatro sítios amostrais, Pinheiral-RJ. Valores com a mesma letra não diferem estatisticamente entre si pelo teste Tukey a $5 \%$.

Figure 2. Averages of accumulated litter $\left(\mathrm{Mg}^{-h a^{-1}}\right)$ and its standard deviation in the four sampling sites, Pinheiral-RJ. Values with the same letter do not differ statistically by Tukey test at $5 \%$. 
Nos tratamentos S1 e S2, os processos de restauração espontânea se verificam entre seis e dez anos, ou seja, a espécie colonizadora espontânea está entrando e apresenta algumas poucas espécies arbustivas.

O maior estoque de serrapilheira acumulada presente no S4 pode ser justificado pela tipologia mais densa, diversa e estratificada da vegetação (estratos arbóreo, arbustivo e herbáceo), além da presença de lianas. Todos esses fatores aumentam a produção de matéria orgânica, conforme observado por alguns autores (Borém \& Ramos, 2002; Correia \& Andrade, 2008), que relacionaram maior diversidade de espécies e densidade com acúmulo de serrapilheira. A relação aporte de serrapilheira e velocidade de decomposição também interferem na camada de biomassa vegetal acumulada (Pagano, 1989).

No presente estudo, as espécies espontâneas são nativas e os ambientes, fisicamente similares, o que permite melhor vínculo entre as espécies dos diferentes estágios sucessionais e seu relacionamento entre produção e retenção de umidade na serrapilheira.

A ausência de diferença significativa entre S3 e S4 indica que a evolução atingida pelo S3, em relação ao acúmulo de serrapilheira, em 15 anos, é próxima das condições de S4 (30 anos), representando um bom indicativo para estabilização da superfície dos solos e influenciando, consequentemente, na restauração florestal espontânea. Segundo Ewel (1976), a serrapilheira acumulada favorece processos particularmente importantes na restauração da fertilidade do solo nas áreas em início de sucessão ecológica, proporcionando, assim, condições favoráveis para o estabelecimento de espécies de níveis sucessionais avançados.

Em relação aos sítios 2 e 3, a ausência de diferença significativa no acúmulo de serrapilheira possivelmente seja atribuída à similaridade dos processos ecológicos envolvidos na restauração, que apresentam variações fisionômicas pequenas, mas, provavelmente, aspectos funcionais similares, considerando-se que ambos os sítios se encontram no estágio inicial de sucessão ecológica. A predominância da Clidemia urceolata nos sítios $2 \mathrm{e}$ 3 contribuiu para o acúmulo da serrapilheira, sendo também observada maior quantidade de material dessa espécie no seu fracionamento por tipo de material (folhas, galhos, casca e frutos, entre outros) (Figura 3). No S1, foram encontrados apenas colmos e hastes de gramíneas.

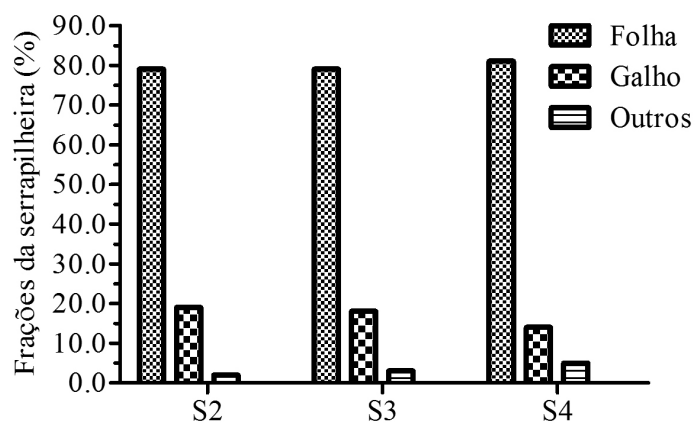

Figura 3. Valores percentuais das frações (folha, galho e outros) da serrapilheira acumulada nos sítios 2, 3 e 4, Pinheiral-RJ.

Figure 3. Percentages of the fractions (leaf, branch and others) of litter accumulated on sites 2 , sites 3 and site $4 \mathrm{~s}$, Pinheiral-RJ.

Apesar da diferença não significativa da serrapilheira acumulada entre S1 e S2, se observou que, a partir de (S2), houve aumento do acúmulo de serrapilheira de espécies diferentes. Segundo Silveira et al. (2007), a camada de serrapilheira acumulada é um fator condicionante para a manutenção da fertilidade do solo e a sustentação do ecossistema, por constituir parte do processo de transferência de água e nutrientes da fitomassa para o solo.

O menor acúmulo de serrapilheira em S1 esta relacionado aos intensos usos no passado e ao seu baixo estado de conservação. Essa serrapilheira é composta por folhas (folhas, colmos e hastes), sendo material leve e carreável pelos ventos e chuvas. Segundo Larcher (2004), leguminosas e gramíneas possuem baixa relação $\mathrm{C}: \mathrm{N}$, determinando a velocidade de decomposição dos tecidos vegetais; entretanto, o colmo e as hastes do capim-colonião são lignificados e demoram a decompor-se, prevalecendo estruturas lignificadas, pobres e poucas folhas.

\subsection{Retenção hídrica}

As maiores retenções hídricas da serrapilheira acumulada foram encontradas nas capoeiras com predominância da espécie Clidemia urceolata (S2) - 343,87\% $\pm 49.079 \%$ e $(\mathrm{S} 3)-339,94 \% \pm 62.190 \%$ $(\mathrm{P}<0,001$ e $\mathrm{F}=16,521)$ (Figura 4$)$. 


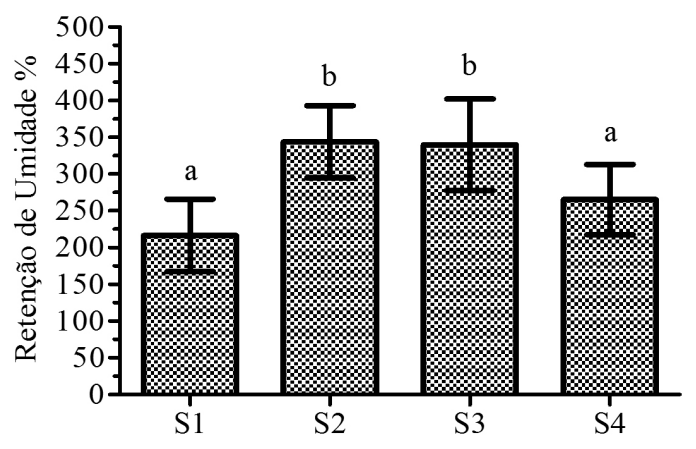

Figura 4. Médias da capacidade de retenção hídrica e seus respectivos desvios padrões dos quatro sítios amostrais, Pinheiral-RJ. Valores com a mesma letra não diferem entre si pelo teste de Tukey a 5\%.

Figure 4. Medium capacity of water retention and their respective standard deviations of the four sampling sites, Pinheiral-RJ. Values with the same letter do not differ by Tukey test at $5 \%$.

A maior capacidade de retenção hídrica da serrapilheira dos sítios 2 e 3 está relacionada à quantidade de folhas da espécie Clidemia urceolata, que apresenta 'aspecto glutinoso' (Matsumoto, 1999), considerando-se este um importante serviço ambiental da espécie na facilitação da restauração florestal espontânea, pois, apesar de menor quantidade de serrapilheira que S4, esta retém umidade sobre a superfície do solo, propiciando condições para germinação, estabelecimento de propágulos e manutenção das plântulas na fase juvenil. Segundo Vallejo (1982), a capacidade de retenção de umidade não depende apenas do peso seco acumulado da serrapilheira, mas das suas características individuais e do tipo e do grau de decomposição do material. A velocidade de decomposição depende do percentual de lignina, polifenóis, carbono, nitrogênio e fósforo, entre outros componentes (Swift et al., 1979). Em ambientes fisicamente similares, esses elementos podem justificar os seus processos de decomposição e ser fontes de preferências ecológicas para espécies colonizadoras.

Blow (1955) encontrou valores entre 200\% e 250\% para serrapilheira acumulada de florestas de carvalho no Tennessee, EUA. Vallejo (1982) obteve valores superiores a $300 \%$, em floresta latifoliada perene no Parque Nacional da Floresta da Tijuca, Rio de Janeiro, valores estes próximos aos encontrados no presente estudo. Essas informações evidenciam a formação de propriedades emergentes nos ecossistemas perturbados e sinalizam sua importância para a evolução do processo de restauração ecológica, pois umidade é fundamental para a germinação, o estabelecimento e o crescimento de plantas.

Entre S1 e S4, observa-se comportamento numérico similar na retenção hídrica da serrapilheira, mas significados distintos na restauração, uma vez que, em S1, pode haver baixa infiltração e perdas por escoamento superficial, conforme observações de campo e também registrado em pastagens em Bananal-SP (Deus, 1991). Em S4, o total de chuva direta precipitada infiltra, abastece a malha de raízes na serrapilheira e nos primeiros centímetros de solo, irrigando a rizosfera em diferentes profundidades desse ecossistema, facilitando o desenvolvimento de espécies exigentes em água e reduzindo os processos erosivos, conforme se observou pela ausência de microsulcos no terreno. A capacidade de retenção hídrica de S1 não se difere estatisticamente de S4; no entanto, as características fitofisionômicas de S1 proporcionam ao solo maior exposição ao impacto das gotas de chuva, ao escoamento superficial e à erosão, causando degradação (Gonçalves et al., 2003).

A serrapilheira desempenha um papel fundamental na regulação dos processos hidrológicos superficiais (Sato, 2008), melhorando a administração de água ao solo, via fluxos subsuperficiais (Oliveira, 1987), disponibilizando água e meios aos indivíduos que irão (re)construir os ecossistemas, e dando celeridade aos processos de restauração.

\section{CONCLUSÕES}

As capoeiras formadas a partir da espécie Clidemia urceolata apresentaram acúmulo de serrapilheira e retenção hídrica diferenciada na fase inicial da restauração florestal espontânea da Mata Atlântica, acumulando menos serrapilheira e retendo mais umidade nos primeiros 15 anos, e aumentando a serrapilheira acumulada e liberando umidade para infiltração e recarga da rizosfera aos 
30 anos, justamente quando as espécies secundárias colonizam o ambiente.

\section{STATUS DA SUBMISSÃO}

Recebido: $24 / 08 / 2012$

Aceito: $16 / 05 / 2013$

Publicado: 30/09/2013

\section{AUTOR(ES) PARA CORRESPONDÊNCIA}

\section{Felipe Araujo Mateus}

Departamento de Ciências Ambientais, Universidade Federal Rural do Rio de Janeiro - UFRRJ, Rod. BR 465, Km 7 , CEP 23890-000, Seropédica, RJ, Brasil e-mail: felipearaujomateus@gmail.com

\section{REFERÊNCIAS}

Blow FE. Quantity and hydrologic characterstics of litter under upland oak forests in Eastern Tennessee. Journal of Forestry 1955; 53: 190-195.

Borém RAT, Ramos DP. Variação estacional e topográfica de nutrientes na serapilheira de um fragmento de mata atlântica. Revista Cerne 2002; 8(2): 042-059.

Brun EJ, Schumacher MV, Vaccaro S, Spathelf P. Relação entre a produção de serapilheira e variáveis meteorológicas em três fases sucessionais de uma floresta estacional decidual no Rio Grande do Sul. Revista Brasileira de Agrometeorologia 2001; 9(2): 277285.

Carvalho PER. Técnicas de recuperação e manejo de áreas degradadas. In: Galvão APM. Reflorestamento de propriedades rurais para fins produtivos e ambientais: um guia para ações municipais e regionais. Brasília: Embrapa; 2000.

Cianciaruso MC, Pires JSR, Delitti WBC, Silva EFLP. Produção de serapilheira e decomposição do material foliar em um cerradão na Estação Ecológica de Jataí, município de Luiz Antônio, SP, Brasil. Acta Botanica Brasilica 2006; 20(1): 49-59. http://dx.doi.org/10.1590/ S0102-33062006000100006

Coelho Netto AL. Evolução de Cabeceiras de Drenagem no Médio Vale do Rio Paraíba do Sul (SP/RJ): Bases para um Modelo de Formação e Crescimento da Rede de Canais sob Controle Estrutural. Revista Brasileira de Geomorfologia 2003; 4(2): 118-167.

Correia MEF, Andrade AG. Formação de serapilheira e ciclagem de nutrientes. In: Santos GA, Silva LS, Canellas LP, Camargo FAO. Fundamentos da matéria orgânica do solo - ecossistemas tropicais \& subtropicais. Metrópole; 2008.

Deus E. O papel da escavação das formigas do gênero Atta na hidrologia de encostas e áreas de pastagem - Bananal (SP) [dissertação]. Rio de Janeiro: Instituto de Geociências, Universidade Federal do Rio de Janeiro; 1991.

Ewel JJ. Litter fall and leaf decomposition in a tropical forest succession in eastern Guatemala. Journal of Ecology 1976; 64: 293-308. http://dx.doi. org/10.2307/2258696

Facelli JM, Facelli E. Interactions after death: plant litter controls priority affects in a sucessional plant community. Oecologia 1993; 95: 277-282. http://dx.doi. org/10.1007/BF00323500

Facelli JM, Pickett STA. Plant litter: its dynamics and effects on plant community structure. The Botanical Review 1991; 57: 1-32. http://dx.doi.org/10.1007/ BF02858763

Figueiredo PHA, Miranda CC, Mateus FA, Valcarcel R. Avaliação do potencial seminal da Cecropia Pachystachya Trécul no banco de sementes do solo de um fragmento florestal em restauração espontânea na Mata Atlântica, Pinheiral - RJ. Revista Biociências da Universidade de Taubaté 2011; 17(2): 43-51.

Gonçalves JLM, Nogueira LR Jr, Ducatti F. Recuperação de solos degradados. In: Kageyama PY, Oliveira RE, Moraes LFD, Engel VL, Gandara FB. Restauração ecológica de ecossistemas naturais. Botucatu: FEPAF; 2003.

Instituto Brasileiro de geografia e Estatística - IBGE. Manual Técnico da Vegetação Brasileira. Manuais Técnicos em Geociências. Rio de Janeiro: IBGE, Diretoria de Geociências, Coordenação de Recursos Naturais e Estudos Ambientais; 2012.

Instituto Nacional de Meteorologia - INMET. Ministério da Agricultura e Reforma Agrária. Normais Climatológicas (1961-1990). Brasília: SPI/ EMBRAPA, 1992

Larcher W. Ecofisiologia vegetal. São Carlos: Rima; 2004.

Matsumoto K. A família Melastomataceae Juss. nas formações campestres do município de Carrancas, Minas Gerais [dissertação]. Campinas: Instituto de Biologia, Universidade Estadual de Campinas; 1999.

Meguro M, Vinueza GN, Delitti WBC. Ciclagem de nutrientes minerais na Mata Mesófila secundária. Produção e conteúdo de nutrientes minerais do folhedo. Boletim de Botânica da Universidade de São Paulo 1979; 7: 61-67.

Menezes CEG. Integridade de paisagem, manejo e atributos do solo no Médio Vale do Paraiba do Sul, Pinheiral-RJ [tese]. Seropédica: Instituto de Agronomia, Universidade Federal Rural do Rio de Janeiro; 2008. 
Miranda CC. Funcionalidade de núcleos de Clidemia urceolata DC. na recuperação de ambientes perturbados da Mata Atlântica [tese]. Seropédica: Instituto de Florestas, Universidade Federal Rural do Rio de Janeiro; 2012.

Miranda CC, Couto WH, Valcarcel R, Nunes-freitas AFN, Francelino MR. Avaliação das preferências ecológicas Clidemia urceolata DC. em ecossistemas perturbados. Revista Arvore 2011; 35(5): 1135-1144. http://dx.doi.org/10.1590/S0100-67622011000600019

Mitchell HH, Teel MR. Winter annual cover crops for no tillage corn production. Journal Madison 1977; 69: 569573.

Oliveira RR. Produção e decomposição de serapilheira no Parque Nacional da Tijuca, RJ [dissertação]. Rio de Janeiro: Instituto de Geociências, Universidade Federal do Rio de Janeiro; 1987.

Pagano SN. Produção de folhedo em mata mesófila semidecídua no município de Rio Claro, SP. Revista Brasileira de Biologia 1989; 49(3): 633-639.

Pires LA, Britez RM, Martel G, Pagano SN. Produção, acúmulo e decomposição da serapilheira em uma restinga da Ilha do Mel, Paranaguá, PR, Brasil. Acta Botanica Brasilica 2006; 20(1): 173-184. http://dx.doi. org/10.1590/S0102-33062006000100016

Portes MCGO, Koehler A, Galvão F. Variação sazonal de deposição de serapilheira em uma Floresta Ombrófila Densa Altomontana no morro do Anhagava- PR. Revista Floresta 1996; 26(1/2): 3-10.
Sato AM. Respostas Geo-Hidroecológicas Relacionadas à Substituição de Pastagens por Plantações de Eucalipto no Médio Vale do Rio Paraíba do Sul: a interface biotasolo-água [dissertação]. Rio de Janeiro: Instituto de Geociências, Universidade Federal do Rio de Janeiro; 2008.

Silveira ND, Pereira MG, Polidoro JC, Tavares SRL, Mello RB. Aporte de nutrientes e biomassa via serrapilheira em sistemas agroflorestais em Paraty (RJ). Revista Ciência Florestal 2007; 17(2): 129-136.

Swift MJ, Heal OW, Anderson JM. Decomposition in Terrestrial Ecosystems. Oxford: Blackwell Scientific Publications; 1979. v. 5 , p. 372.

Toledo LO, Pereira MG. Dinâmica da deposição de serrapilheira em florestas secundárias do município de Pinheiral, RJ. Revista Floresta e Ambiente 2004; 11(1): 3946.

Valcarcel R, Silva ZS. A eficiência conservacionista de medidas de recuperação de áreas degradadas: proposta metodológica. Revista Floresta 2000; 21(1-2): 101-114.

Vallejo LR. A influência do Litter na distribuição das águas pluviais [dissertação]. Rio de Janeiro: Universidade Federal do Rio de Janeiro; 1982.

Voigt VP, Walsh RPD. Hidrologische prozesse in bodenstreu. Schriften des Naturwissenschaftlichen Vereins fur Schleswig-Holstein 1976; 46: 35-54.

Zar JH. Biostatistical analysis. 4th ed. New Jersey: Prentice-Hall; 1999. 